Proc. of the Seventh International Conference On Advances in Computing, Electronics and Electrical Technology - CEET 2017. Copyright (C) Institute of Research Engineers and Doctors. All rights reserved.

ISBN: 978-1-63248-126-9 doi: 10.15224/ 978-1-63248-126-9-30

\title{
RLG Dither Removal Using Wavelet Transforms
}

\author{
Bharath Regimanu, Kakarla Subba Rao, Kishore Chandra Das, Ch.Raja Kumari, P.Neeharika
}

\begin{abstract}
Ring Laser Gyroscopes (RLGs) are widely used in many airborne and navigation systems for accurate measurement of the true rotation of the body movement. But the RLG's suffer a serious problem at low frequencies known as Lock -in frequency. To avoid lock-in problem, the RLG is vibrated mechanically to a high frequency which is known as Dithering. In order to get the true rotation of the body the dither signal has to be removed. Single stage, multistage and multirate filters are suggested to remove the dither signal. These filters have the disadvantage that either the FIR filter length is too large or the phase characteristics are not linear. In this work multiresolution techniques using Wavelet Transforms (WTs) are used to remove the dither signal. Six level multi resolution analysis is carried out with various types of wavelets like Discrete Meyer and Daubechies 45 (db45) etc. With none of the standard wavelets, the original and reconstructed signals are matched. A new wavelet is designed to remove the dither signal. The required signal can be constructed back using the approximation coefficients at level 6 . The dither signal is attenuated by $265 \mathrm{~dB}$, and the phase characteristics are found to be linear in the pass band. The computational complexity is also less compared to the three stage combined filter reported earlier.
\end{abstract}

Keywords-Ring Laser Gyroscope, Multiresolution,

\section{I.INTRODUCTION}

Gyroscope is basically a rotation sensor which is used to measure the absolute angular rotation of any rotating system. This instrument is an essential requirement for navigation and control of a moving vehicle. The advantage of RLG is that it is less sensitive to environmental conditions and its performance does not depend on gravity of the earth ' $\mathrm{g}$ '. It is also less sensitive to thermal conditions and magnitude fields. Hence it is more accurate and more stable.

Bharath Regimanu, Senior Research Assistant, Dept. of ECE

Chaitanya Bharathi Institute of Technology, Gandipet, Hyderabad, 500075 India.

Kakarla Subba Rao, Professor, Dept. of ECE

Chaitanya Bharathi Institute of Technology, Gandipet, Hyderabad, 500075 India

Kishore Chandra Das, Scientist F,

Research Centre Imarat(DRDO), Vignyana Kancha, Hyderabad 500069 India.

Ch,Raja Kumari, Asst. Prof, Dept. of ECE

VNRVJIET, Bachupally, Hyderabad, 500090

India.

P Neeharika, PG Student, Dept. of ECE

Chaitanya Bharathi Institute of Technology, Gandipet, Hyderabad, 500075 India.
The RLG is essentially an interferometer with an active laser medium in the ring cavity to generate two propagating laser $\mathrm{w}$ aves-one in clockwise $(\mathrm{CW})$ and the other in counter clockwis e (CCW) within the cavity as shown in fig.(1).

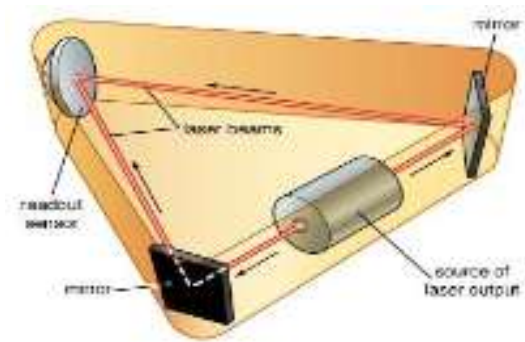

Figure.(1).Ring Laser Gyroscope

A reflector prism is used to collate the counter propagating beams to produce a stationary spatial interference pattern. The $\mathrm{CW}$ and CCW path lengths are same when no rotation is appli ed. When the laser cavity rotates a path difference $\Delta L$ exits bet ween the two counter propagating waves and this is proportion al to the difference in frequency which is called the beat frequ ency[1]. The beat frequency $\Delta f$ is proportional to the rate of a ngular rotation as given in equation.

$$
\begin{gathered}
\Delta f \alpha \Omega \\
\text { or } \Delta f=K \Omega
\end{gathered}
$$

where $\Omega$ the rate of rotation along the sensitive axis and $\mathrm{K}$ is a constant that depends on the path the RLG length, area of the ring cavity and wavelength of the laser source. When $\Delta \mathrm{f}$ is bel ow a certain threshold value called lock-in state due to the mut ual coupling of the waves, $\Delta \mathrm{f}$ becomes zero and the RLG outp ut vanishes. To avoid the lock-in state, the RLG is vibrated me chanically in both $\mathrm{CW}$ and $\mathrm{CCW}$ directions to a high oscillatin $\mathrm{g}$ frequency which is called 'Dithering'. The dither frequency $\mathrm{f}$ or a given RLG is constant which is in the range of (350-500) $\mathrm{Hz}$ and the magnitude of the Dither signal (mechanical vibrati on) is about $10^{4}$ to $10^{5}$ times higher than the actual rotation rat e of the body. In order to get the true rotation rate of the body, the Dither signal is to be removed[5]. In a typical RLG with a dither frequency of $500 \mathrm{~Hz}$ and an amplitude of $100 \mathrm{arc}-\mathrm{sec}$, th e dither angular rate is about $90 \% \mathrm{sec}$. Two types of dither rem oval techniques i) the dither stripping technique and ii) the us e of digital filters which is relatively a new concept are used.

Both FIR and IIR filters are reported in the literature to rem ove the dither signal. But neither a single stage FIR nor a sing le stage IIR filter provide the necessary attenuation. Recently $t$ hree stage combined filters (FIR\&IIR) are suggested. But the phase characteristics of IIR filters are not linear. Though the at tenuation achieved is above $140 \mathrm{~dB}$, the phase characteristics a re not linear in the pass band and also the computational comp 
Proc. of the Seventh International Conference On Advances in Computing, Electronics and Electrical Technology - CEET 2017. Copyright ( $)$ Institute of Research Engineers and Doctors. All rights reserved.

ISBN: 978-1-63248-126-9 doi: 10.15224/ 978-1-63248-126-9-30

lexity is high[7].

\section{Wavelet Transform(WT)}

WT is used in many signal processing applications such as vid eo compression, internet communications compression, object recognition etc. It can efficiently represent some signals, espec ially ones that have localised changes. WT can also be used in multiresolution analysis.

Even though many advanced signal processing algorithms are developed, Fourier Transform (FT) is still the basic and power ful tool even today. This is mainly for two reasons. Firstly, the FT can be efficiently implemented using FFT and secondly the FT is an eigen function of a linear time invariant system. In FT the basis function, $e^{-j 2 \pi f t}$, exists from minus infinity to plus $\mathrm{i}$ nfinity and the integration is over the entire time period. As a $\mathrm{r}$ esult the time information is lost and the FT gives only the glo bal characteristics of the signal. Hence it is not suitable for the analysis of non stationary signals. But ideally in many applicat ions we would like to compute the instantaneous FT or FT ove $r$ a finite interval of time. The Short Time Fourier Transform ( STFT) gives the local FT. By shifting the window in the time domain one can get the Fourier coefficients as a function of ti me and frequency.

The STFT of a signal $\mathrm{x}(\mathrm{t})$ is given by

$X(\tau, F)=\operatorname{STFT}\left(x(t)=\int_{-\infty}^{+\infty} x(t) W(t-\tau) e^{-j 2 \pi f t} d t\right.$

where $W(t-\tau)$ is the window shifted by $\tau$ units.

The frequency content of the signal around time ' $\tau$ ' is given by $X(\tau, F)$. The fixed length window is the main limitation in ST FT in the time-frequency resolution. For example, for analysin $\mathrm{g}$ transient like signals (high frequency) the window length sh ould be small and for analysing steady state signals (low frequ ency), the window length should be large. In WT, the length o $\mathrm{f}$ the window is selected depending on the frequency of the si gnal. In WT the signal is decomposed into a series of basis fun ctions. All the basis functions are derived from a single parent wavelet by means of two operations -Dilation/ stretch and tran slation /shift. The wavelet function must have finite duration a nd for the inverse WT to exist, the total area under the parent wavelet must be equal to zero.

WT can also be viewed in terms of the band pass filters. An octave is a level of resolution, where each octave can be envisi oned as a pair of FIR filters as shown in fig.(2).

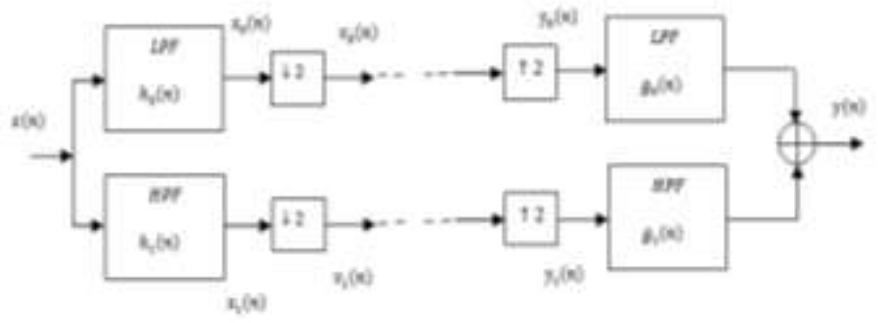

$\begin{array}{ll}\text { (a)Analysis filters } & \text { (b)Synthesis filters }\end{array}$

Figure.(2): One octave wavelet analysis

The down samplers and the up samplers help to reduce the re dundancy in the data and to make the transform efficient.

The LPF produces the average signal where as the HPF produ ces the detail signal.

\section{II. a) Wavelet Analysis}

We know that $X_{0}(Z)=X(Z) H_{0}(Z)$ and

$$
X_{1}(Z)=X(Z) H_{1}(Z)
$$

The outputs of the down samplers are

$$
\begin{aligned}
V_{0}(Z) & =\frac{1}{2}\left[X\left(Z^{\frac{1}{2}}\right) H_{0}\left(Z^{\frac{1}{2}}\right)+X\left(-Z^{\frac{1}{2}}\right) H_{0}\left(-Z^{\frac{1}{2}}\right)\right] \\
\text { and } V_{1}(Z) & =\frac{1}{2}\left[X\left(Z^{\frac{1}{2}}\right) H_{1}\left(Z^{\frac{1}{2}}\right)+X\left(-Z^{\frac{1}{2}}\right) H_{1}\left(-Z^{\frac{1}{2}}\right)\right]
\end{aligned}
$$

The outputs of the up samplers are

$$
Y_{0}(Z)=V_{0}\left(Z^{2}\right)=\frac{1}{2}\left[X(Z) H_{0}(Z)+X(-Z) H_{0}(-Z)\right]
$$

and $Y_{1}(Z)=V_{1}\left(Z^{2}\right)=\frac{1}{2}\left[X(Z) H_{1}(Z)+X(-Z) H_{1}(-Z)\right]$

The output signal $Y(Z)$ of the synthesis filter bank is given by

$$
\begin{aligned}
Y(Z)=\frac{1}{2}\left[G_{0}(Z) H_{0}(Z)+G_{1}(Z) H_{1}(Z)\right] X(Z) \\
+\frac{1}{2}\left[G_{0}(Z) H_{0}(-Z)+G_{1}(Z) H_{1}(-Z)\right] X(-Z)
\end{aligned}
$$

In order to get perfect reconstruction, one can choose

$H_{1}(Z)=H_{0}(-Z), \quad G_{0}(Z)=-H_{1}(-Z), \quad$ and

$G_{1}(Z)=-H_{1}(Z)$

then $y(n)=x(n-l)$

\section{II. b) Continuous Wavelet Transform (CWT)}

The CWT of the signal $x(t)$ is given as

$$
W_{x}(a, \tau)=\int_{-\infty}^{\infty} x(t) \sqrt{a} \varphi(a(t-\tau)) d t
$$

where $\varphi(t)$ is the wavelet function shifted by $\tau$ and scaled by ' $a$ '.

$$
W_{x}(a, \tau)=<x(t), \sqrt{a} \varphi(a(t-\tau))>
$$

The CWT can be expressed as the inner product as in eqn.(4) The inner product can be computed by using filters. The wavel et function is given as

$$
\varphi(t)=\sqrt{2} \sum_{k} g(k) \emptyset(2 t-k)
$$

And the scaling function $\varnothing(t)$ is determined through recursivel $\mathrm{y}$ applying the filter coefficients and is given by

$$
\varnothing(t)=\sqrt{2} \sum_{k} h(k) \varnothing(2 t-k) \quad \text { wher }
$$

$\mathrm{e} h(\mathrm{k})$ is the impulse response of the low pass filter and $\mathrm{g}(\mathrm{k})$ is the impulse response of the high pass filter. Both $\mathrm{h}(\mathrm{k})$ and $\mathrm{g}(\mathrm{k})$ are of finite length.

\section{II. c) Discrete Wavelet Transform (DWT)}

A unique relation exists between the parameters of a class of $p$ erfect reconstruction octave band QMF bank (fig.2) and DWT. The scaling function allows us to have an approximation at the last level of resolution; the initial level of resolution having th e input signal itself. After J levels of resolution, the result of th e scaling function on the signal is zero. The scaling function or the lowpass filter output gives the approximation of the signal as given in eqn.(5). 
Proc. of the Seventh International Conference On Advances in Computing, Electronics and Electrical Technology - CEET 2017. Copyright ( $)$ Institute of Research Engineers and Doctors. All rights reserved.

ISBN: 978-1-63248-126-9 doi: 10.15224/ 978-1-63248-126-9-30

$A(j, n)=\sum_{m=0}^{N-1} A(j-1, m) h(2 n-m), \quad \mathrm{j}=0,1,2 \ldots(\mathrm{J}-1)$

where $\mathrm{j}$ is the current octave (level) and

$\mathrm{J}$ is the total numbers of octaves

$\mathrm{n}$ is the shift in time

The term (2n-m) incorporates scaling resulting in half the outp uts for octave $\mathrm{j}$ compared to octave $\mathrm{J}-1$.

and $\mathrm{A}(0, \mathrm{n})=\mathrm{x}(\mathrm{n})$

The wavelet function or the HPF output gives us the detail si gnal which can be recursively generated using eqn.(7)

$$
D(j, n)=\sum_{m=0}^{N-1} A(j-1, m) g(2 n-m)
$$

\section{II. d) Multiresolution}

In this work multi resolution Wavelet Transform ( WT ) techni ques are used to remove the dither signal. Multiresolution is th e process of taking the output from one channel and putting it $t$ hrough another pair of analysis filters. Specifically, the outpu t of LPF after down sampling at level 1 is passed through ano ther pair of filters which produces the average and details at $t$ he next level as shown in fig.(3).

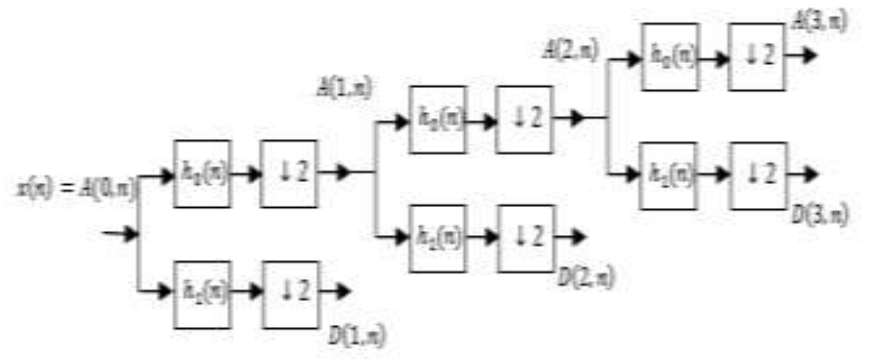

Figure.3. Three level resolution analysis filter bank

Figure shows three levels of resolution of analysis filters. The approximation coefficients $A(j, n)$ and the detail coefficients at each level are computed using eqns.(5) and (7).

Fig.(4) shows the corresponding frequency division in each level [12]. The band width of the LPF at level 3 is 0 to $\pi / 8$ and that of HPF is $\pi / 8$ to $\pi / 4$.

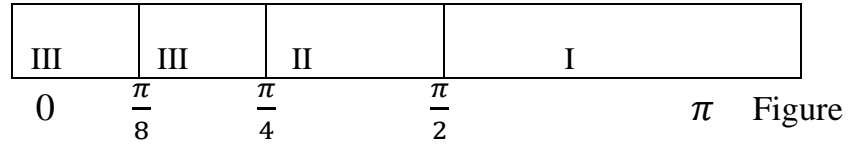

.4.Frequency bands for three level analysis filter bank.

Fig. (5) shows three level synthesis filter bank which is used $t$ o reconstruct the signal back. The scaling function output at le vel 3 and the wavelet function outputs at all 3 levels are requir ed to reconstruct the signal back as given in eqn.(8). That is on ly $D(j, n), j=1$ to 3 and $A(3, n)$ are required to be stored to ge $\mathrm{t}$ the signal $\mathrm{x} 1(\mathrm{n})$ back.

$$
\begin{aligned}
A(j, k)=\sum_{m=0}^{N-1} g_{0}(2 k-m) A(j+1, m) & \\
& +\sum_{m=0}^{N-1} g_{1}(2 k-m) D(j+1, m)
\end{aligned}
$$

Let $g_{0}(n)$ and $g_{1}(n)$ be the corresponding scaling and wavelet $f$ unctions for the synthesis filter bank. $g_{0}(n)$ and $g_{1}(n)$ can be fo und from $\mathrm{h}_{0}(\mathrm{n})$ using eq.(2).

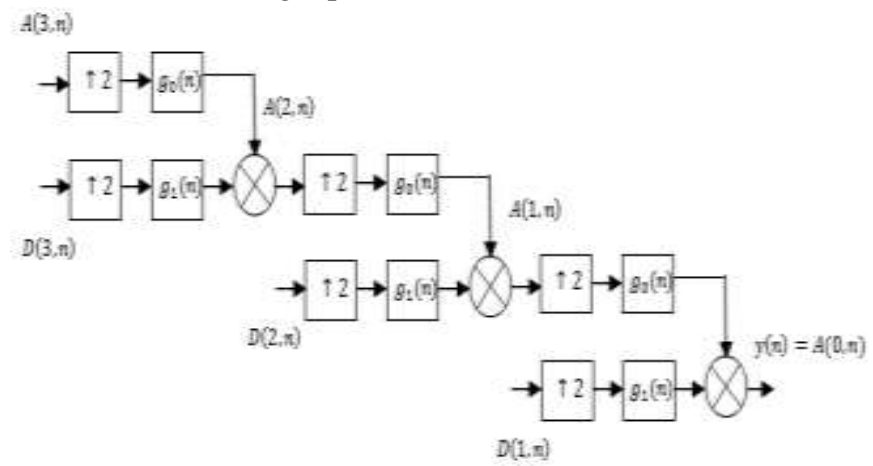

Figure.5. Three level resolution synthesis filter bank.

\section{III) Dither filtering using WT.}

The magnitude of the dither signal is about $10^{4}$ to $10^{5}$ times higher compared to the true rotation angle. The pass band is from to $70 \mathrm{~Hz}$. The specifications of the signal to be filtered are given in table.

\begin{tabular}{|l|c|}
\hline Pass band & (0 to 70$) \mathrm{Hz}$ \\
\hline Stop band & (90 to 5000$) \mathrm{Hz}$ \\
\hline Dither band & $400 \mathrm{~Hz}$ \\
\hline Attenuation in pass band & $<3 \mathrm{~dB}$ \\
\hline Attenuation in stop band & $>100 \mathrm{~dB}$ \\
\hline Attenuation in dither band & $\geq 160 \mathrm{~dB}$ \\
\hline Sampling freqency & $10 \mathrm{KHz}$ \\
\hline
\end{tabular}

Table 1. Specifications of the filter to be designed.

A synthetic RLG signal is generated using the MATLAB com mand.

$$
\begin{array}{rl}
x(t)= & a 1 * \sin (2 * p i * f p 1 * t)+a 2 * \sin (2 * p i * f p 2 * t)+ \\
\text { a } 3 & * \sin (2 * p i * f p 3 * t)+a 4 * \sin (2 * p i * f p 4 * t)+ \\
& \mathrm{b} 1 * \sin (2 * p i * f s 1 * t)+b 2 * \sin (2 * p i * f s 2 * t)+ \\
& \mathrm{d} * \sin (2 * p i * f d * t)
\end{array}
$$

where $\mathrm{a} 1=0.000003, \mathrm{a} 2=0.000008, \mathrm{a} 3=0.000004, \mathrm{a} 4=0.00000$

$4, \mathrm{~b} 1=0.0000002, \mathrm{~b} 2=0.000002$, and $\mathrm{d}=10$

$\mathrm{fp} 1=5 \mathrm{~Hz}, \mathrm{fp} 2=20 \mathrm{~Hz}, \mathrm{fp} 3=35 \mathrm{~Hz}, \mathrm{fp} 4=55 \mathrm{~Hz}$ (pass band frequen cies), fs $1=95 \mathrm{~Hz}, \mathrm{fs} 2=120 \mathrm{~Hz}$ (stop band frequencies) and $\mathrm{fd}=4$ $00 \mathrm{~Hz}$ (dither frequency)

The input signal $x(t)$ is plotted in fig.(6) and its magnitude sp ectrum is plotted in fig.(7). From the figures it may be observe $\mathrm{d}$ that only $400 \mathrm{~Hz}$ frequency is present and the other frequenc ies are not visible. The magnitude of the Fourier spectrum at $t$ he dither frequency $(400 \mathrm{~Hz})$ can be measured as $2.6^{*} 10^{+4}$ 
Proc. of the Seventh International Conference On Advances in Computing, Electronics and Electrical Technology - CEET 2017. Copyright ( $)$ Institute of Research Engineers and Doctors. All rights reserved.

ISBN: 978-1-63248-126-9 doi: 10.15224/ 978-1-63248-126-9-30

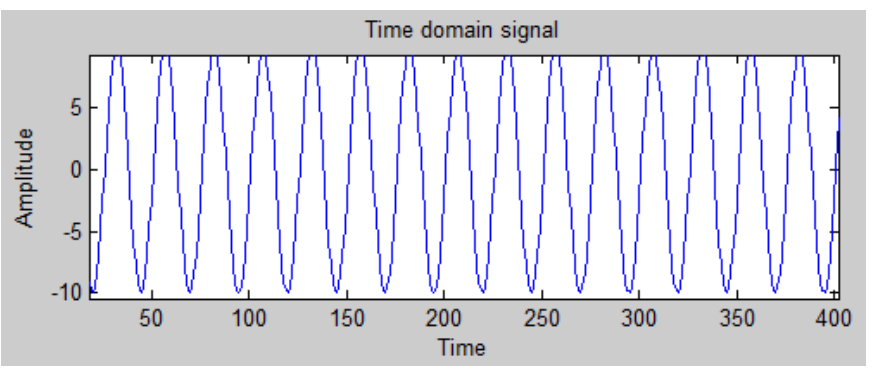

Figure.6. Input signal $\mathrm{x}(\mathrm{t})$ in time domain

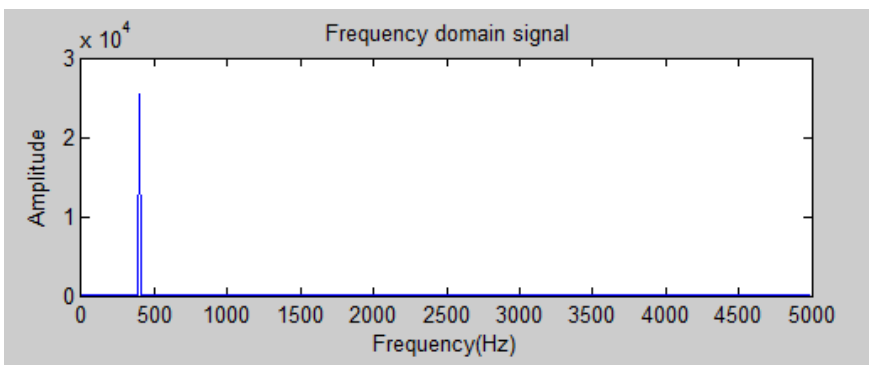

Figure.7. Magnitude spectrum of $x(t)$ in frequency domain.

The signal $\mathrm{x}(\mathrm{t})$ is processed using the concept of multi resol ution. As shown in fig.(3), at each octave certain band of frequ encies are eliminated. Hence analysis filter bank can also be us ed as filter to remove the unwanted frequencies. Since the freq uencies to be rejected are from $90 \mathrm{~Hz}$ to $5 \mathrm{KHz}$, six level resolu tion analysis is used. The frequency division diagram for the $\mathrm{e}$ ntire frequency range is shown in fig.(8). The sixth level allo ws the frequencies from ( 0 to 78$) \mathrm{Hz}$ which is the required pass band and all other frequencies are rejected[13]. Hence the outp ut at level six gives the required filtered signal.

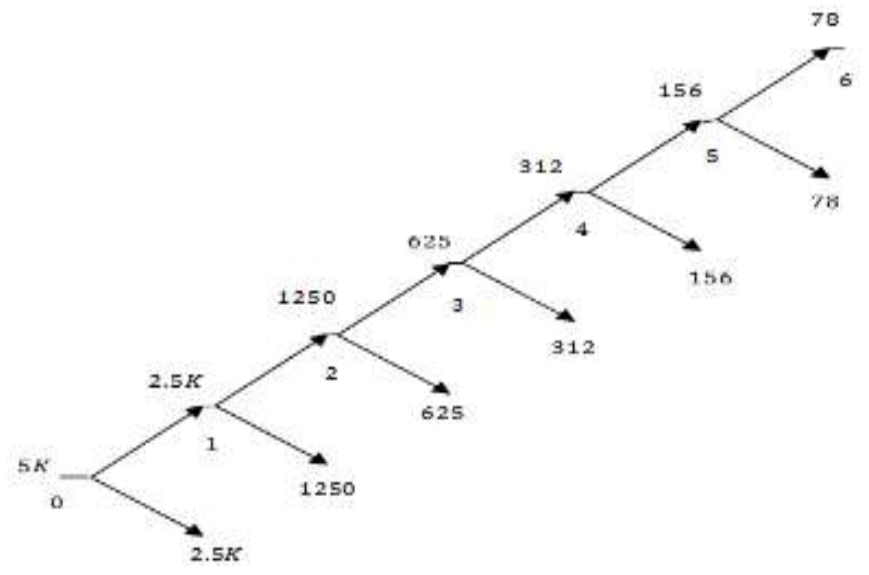

Figure.8. Six level Frequency division diagram .

Various types of orthogonal wavelets namely discrete Meyer a nd Daubechies 45 (db45) etc. are used to remove the dither sig nal. The Discrete Meyer wavelet and the corresponding scalin $\mathrm{g}$ function are shown in Fig.(9).
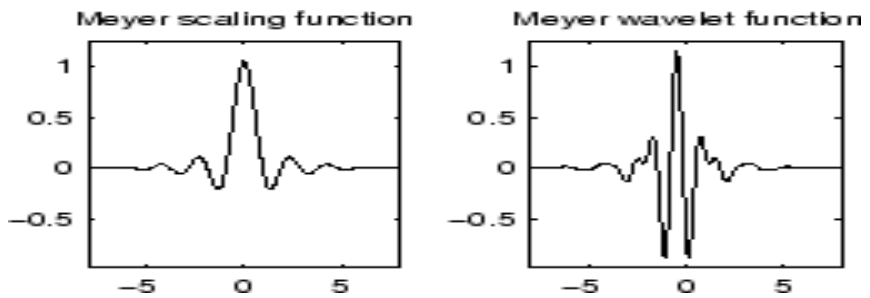

Figure.(9). Meyer scaling and wavelet function.

None of the standard wavelets are found to be suitable to remo ve the dither. Either some spurious frequencies are present in $t$ he spectrum or the reconstructed time signal is different from $t$ he original signal. Hence a new wavelet function is designed $t$ o remove the dither signal completely without distorting the or iginal signal.

\section{IV) Design of new Wavelet for dither removal}

Firstly a FIR lowpass filter is designed to meet the given speci fications. The best window is found to be Kaiser window and $t$ he optimal length of the filter is found to be 35 . Let $h_{0}(n)$ be $t$ he impulse response of the low pass filter or the scaling functi on and the impulse response of the HPF or the wavelet functi on be $h_{1}(n)$. From wavelet theory we know that

$$
\mathrm{h}_{1}(\mathrm{n})=(-1)^{\mathrm{n}} \mathrm{h}_{0}(\mathrm{n})
$$

The following properties are satisfied for the new wavelet fu nction.

$$
\begin{aligned}
& <h_{0}(x) \cdot h_{0}(n)>=1 \\
& <h_{0}(x) \cdot h_{1}(n)>=0 \\
& <h_{1}(x) \cdot h_{1}(n)>=1
\end{aligned}
$$

The scaling function and the wavelet functions are orthogonal and normal or orthonormal. Both the functions are plotted in $\mathrm{F}$ ig.(10).

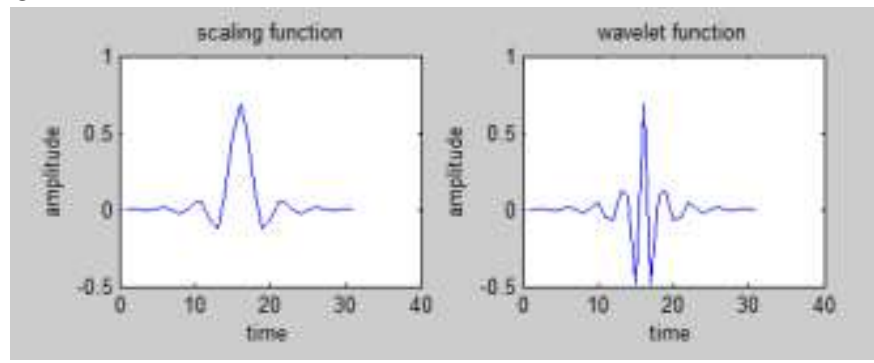

Figure.(10). Scaling and wavelet function of new wavelet.

The frequency responses of the new wavelet, discrete Meyer wavelet and Daubechies 45 are plotted in Fig.(11). It can be ob served that the width of the transition band is less for the new wavelet compared to other wavelets. 
Proc. of the Seventh International Conference On Advances in Computing, Electronics and Electrical Technology - CEET 2017. Copyright ( $\odot$ Institute of Research Engineers and Doctors. All rights reserved.

ISBN: 978-1-63248-126-9 doi: 10.15224/ 978-1-63248-126-9-30

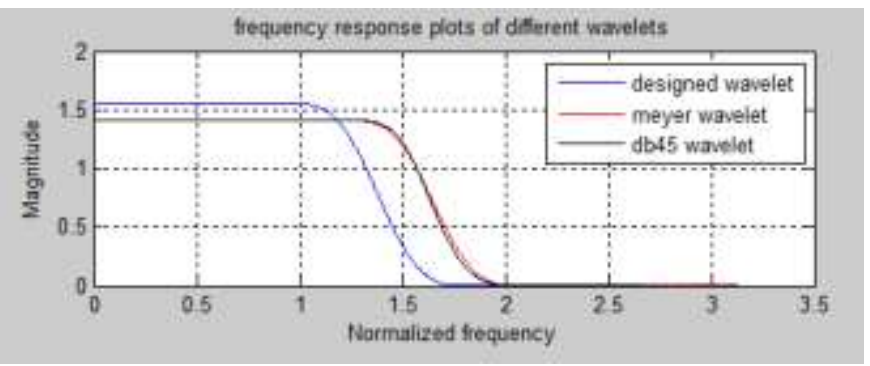

Figure.(11).Plot of Frequency Response

\section{V) Results \& Discussion}

The input signal, $x(t)$, is given as input to the six octave analys is filter bank as shown in fig.(2). Note that fig.(2) shows only a three level tree. Let $h_{0}(n)$ and $h_{1}(n)$ are the scaling and wave let functions. The approximation coefficients at level one are $p$ rocessed again by another pair of filters. The frequency range at level six is 0 to $78 \mathrm{~Hz}$ which is the required pass band signa 1 and all other frequencies including dither are eliminated. The desired signal, $x_{1}(t)$, is reconstructed back using eqn.(8) and $t$ he approximation coefficients at octave 6 , The original signal without dither, $\mathrm{x}_{2}(\mathrm{t})$, is generated using the command

$$
x_{2}(t)=a 1 * \sin (2 * p i * f p 1 * t)+a 2 * \sin (2 * p i * f p 2 * t)+
$$$$
\text { a } 3 * \sin (2 * p i * f p 3 * t)+a 4 * \sin (2 * p i * f p 4 * t) \quad(10)
$$

The signals $\mathrm{x}_{1}(\mathrm{t})$ and $\mathrm{x}_{2}(\mathrm{t})$ are plotted in Fig.(12) and their Fo urier spectra are plotted in Fig.(13). From the figures it is obse rved that there is a perfect match in both the domains. The ma gnitude of the Fourier spectrum at the dither frequency $(400 \mathrm{~Hz}$ ) is found to be $0.1 \times 10^{-8}$ (on zooming Fig.13). It means that $t$ he dither signal is attenuated by about $265 \mathrm{~dB}$. Since the origin al signal contains only four frequencies at $5 \mathrm{~Hz}, 20 \mathrm{~Hz}, 35 \mathrm{~Hz}$ a nd $55 \mathrm{~Hz}$ the spectrum of the reconstructed signal also shows $\mathrm{f}$ our peaks at $5 \mathrm{~Hz}, 20 \mathrm{~Hz}, 35 \mathrm{~Hz}$ and $55 \mathrm{~Hz}$ respectively. This im plies that the new wavelet has passed all the pass band signals (0 to 70$) \mathrm{Hz}$ and attenuated the dither signal as well as the stop band frequencies to the required level.

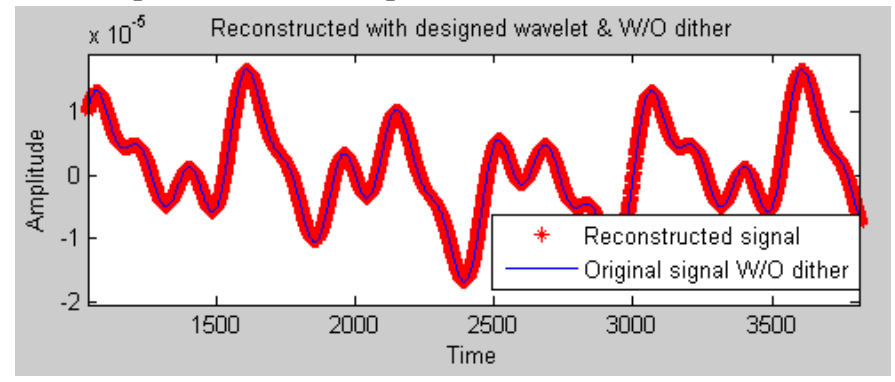

Figure.(12). Plot of Reconstructed signal.

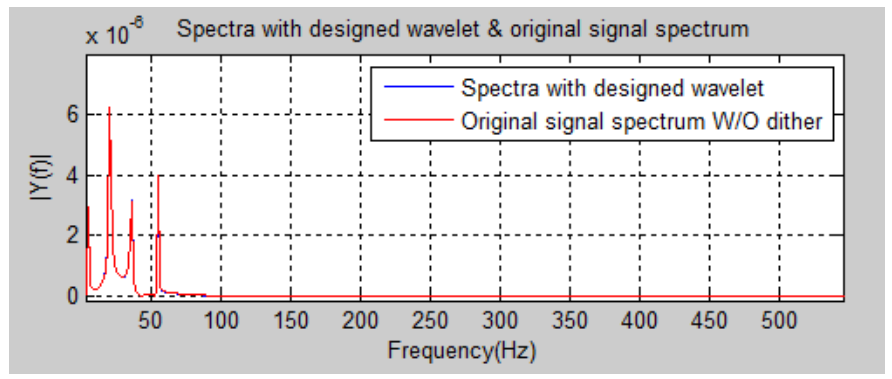

Figure.(13): Fourier spectrum of the reconstructed signal and

\section{VI) Conclusion:}

The principle of multi resolution Wavelet analysis is used to re move the dither signal. The standard wavelets like Discrete $M$ eyer, Daubechies $45(\mathrm{db} 45)$ etc. are found to be not suitable to remove the dither. Hence a new wavelet is designed. The trans ition band width of the new wavelet is lower than the transitio $\mathrm{n}$ band widths of discrete Meyer and db45. The new wavelet a ttenuates the dither signal by about $265 \mathrm{~dB}$. There is a perfect agreement in the original and the reconstructed signals in both time and frequency domains.

VII) Acknowledgements:

The authors are very much thankful to the Director, RCI, Hyd erabad for providing the financial support to carry out the rese arch work.

\section{REFERENES}

[1]. Dr. Frederick Aronowitz, "Fundamentals of the Ring Laser Gyro", 11430, Manzanita Trail, Dewey, Az 86327, U.S.A

[2]. Neil M Barbour, John $M$ Elwell, Roy $H$ Setterlund, “INERTIAL INSTRUMENTS: WHERE TO NOW?", The Charles Stark Draper Laboratory, Inc. Cambridge, Massachusetts 02139

[3]. Jeng Nan Juang and R. Radharamanan, "Evaluation of Ring Laser and Fiber Optic Gyroscope Technology", School Of Engineering, Mercer University, Macon, GA 31207 USA.

[4]. D. Titterton and J. Weston, "Strapdown Inertial Navigation Technology”, IEEE Radar, Sonar, Navigation and Avionics Series, 2nd Ed. U.K. Michael Faraday House, 2005.

[5]. M. Faucheux, D. Fayoux and J. J. Roland, "The Ring Laser Gyro”, Optics (Paris), 1988, Vol. 19, No 3, Pp. 101-115.

[6]. Richard Choi, "Advanced Gyroscope Technology", Systems Center, San Diego, August 2008.

[7]. K.Subba Rao, KC Das, Arjun M \& Swapna $P$ "Development of multistage digital filter for dither removal in $R L G^{\prime \prime} 2$ 2nd International Conference on recent trends in Signal Processing, Image Processing \&VLSI(ICrtSIV-2015), Bangalore pp(872-880)

[8]. Xi Zhang, "Design of orthogonal graph wavelet filter" IECON 2016-42nd Annula Conference of the IEEE Industrial Electronics Society. Page(s):889 - 894

[9]. V.Vijendra, Meghana Kulkarni, "ECG signal filtering using DWT harr wavelets coefficient techniques" 2016 International Conference on Emerging Trends in Engineering , Technology and Science(ICETETS), Page(s): $1-6$

[10]. R.Shantha Selva kumara, S.Bharathi, V.Sadasivam, "Design of Optimal Discrete Wavelet for ECG signal using Orthogonal Filter Bank" International Conference on Computational Intelligence and Multimedia Applications(ICCIMA 2007), Volume:1, Pages:525-529

[11].L.G.Akulov, "Wavelet filtering in Digital Signal processing Systems” 2014 International Conference on Actual problem of Electron Devices Engineering (APEDE), Pages:5-6

[12]. Roberto Cristi, "Modern Digital Signal Processing” Thomson Books/cole

[13]. S.K.Mitra, "Digital signal Processing" Tata Mc.Graw-Hill, $3^{\text {rd }}$ edition. 